\title{
7. Koloniales Schreiben zum Sprechen bringen
}

Die Untersuchung der Texte, die um den Fall Hutten produziert wurden, hat als konkretes Beispiel veranschaulicht, wie das Schreiben den Übergang von der >Entdeckung//Eroberung zur Kolonialisierung von Spanisch-Amerika vollzogen und alltägliche koloniale Normalität hergestellt hat. Dabei wurde die zentrale Rolle des kolonialen Apparats in diesem Prozess deutlich. Er konnte als Maschinerie gefasst werden, die über den Mechanismus der Interpellation Subjekte anruft und koloniale Subjekte generiert. Der Begriff Maschinerie soll die Anlage als komplexes und dynamisches System verdeutlichen, an dessen Funktionieren alle involvierten Akteure mitwirken. Indem der koloniale bürokratische Apparat die »Kommunikationsbedingungen für Distanzherrschaft « (Brendecke 2009a: 345) schuf, wurde der Kreis der potentiell mitwirkenden Beteiligten erweitert. Obwohl damit "größere Gruppen und breitere soziale Schichten an der Macht« partizipiert hätten, habe das gemäß Brendecke der Krone den Vorteil verschafft, ein Mittel zur Begrenzung der lokalen Machthaber in der Hand zu haben. Dieser Wechsel der Kolonialpolitik von der Eroberung zur Kolonisierung fand seinen Ausdruck in den Leyes Nuevas von 1542, die zur schleichenden Entmachtung der Eroberer und Kolonisatoren geführt haben. Dieser Herrschaftsverlust leitete eine Phase des Machtvakuums ein, bevor die Führungsrollen neu geordnet wurden. Der >Fall Hutten ist in diesem Machtvakuum zu verorten.

Ich habe dieser Untersuchung die Frage zugrunde gelegt, wie die >kolonialen Texte ihres Korpus produziert wurden, ob es übergreifende, die Generierungsprozesse aller Texte einleitende und antreibende, typisch koloniale Mechanismen gibt und wie sich diese skizzieren lassen. Im Folgenden sollen die wichtigsten Zwischenergebnisse resümiert werden, indem die Beiträge der einzelnen Kapitel zur Beantwortung dieser Fragestellung zusammengetragen werden. 
In der Einleitung wird die Problematik für die Lektüre kolonialer Texte herausgearbeitet, die sich aus der Tilgung des europäischen >Anderen lonialen Diskurs ergibt. Diese Problematik lag auf den ersten Blick durchaus nicht auf der Hand, denn von der Tilgung sind lediglich Spuren zurückgeblieben, die es erst einzukreisen galt. Wie das Beispiel der Bezeichnung »India Magdalena« gezeigt hat, können sich die Spuren zwar an der Textoberfläche manifestieren, sich aber dennoch bei nicht geschärftem Blick als transparent erweisen. Im Zuge einer vertieften Auseinandersetzung mit den verschiedenen Positionen der Kolonialforschung formierte sich die Erkenntnis, dass die Bezeichnung »India« und der Name »Magdalena« die Tilgung des >Anderen produzieren und gleichzeitig bezeugen, indem sie ausschließlich die europäische Perspektive zulassen und die Benutzenden dieser Wörter automatisch in den kolonialistischen Modus zwingen.

Daraus ergibt sich die bange Frage, wie man koloniale Texte lesen kann, ohne sich an der genannten Tilgung zu beteiligen und in den kolonialistischen Diskurs einzustimmen. Ein Ansatz - so eines der Ergebnisse dieser Untersuchung - besteht darin, dass die Produktionsmechanismen der Kolonialität beschrieben und somit fassbar werden. Damit kann ein Bewusstsein für koloniale Fallen geschaffen werden. Dies ist auch bitter nötig: Bei der Darstellung der verschiedenen Positionen der Kolonialforschung in Kapitel 2 haben wir gesehen, dass sogar bei Autoren, die explizit das Anliegen formulieren, nicht der eurozentrischen Perspektive anheimfallen zu wollen, die Gefahr lauert, dass europäische Standards und Beurteilungskriterien der Kolonialforschung als Muster (noch immer!) zugrunde gelegt werden.

Kapitel 1 dient der Orientierung des Lesers, der Leserin, indem der historische Hintergrund des >Falls Hutten $<$ dargelegt wird. Dabei spielt die Augsburger Handelsgesellschaft der Welser eine zentrale Rolle, da sie von der Krone per Konzession beauftragt wurde, das Land zu erschließen. Die Welser und ihre Vertreter fungieren als eine Art Intermediär in der Hierarchie zwischen den Machthabern vor Ort und der Krone. Dies äußert sich zum Beispiel in der Auseinandersetzung zwischen Carvajal und Hutten insofern, als Letzterer sich in erster Linie gegenüber den Welsern und erst in zweiter Linie gegenüber dem König verpflichtet sieht. Doch die Machtstellung der Welser und damit auch ihrer Repräsentanten vor Ort beginnt just in jenem Moment zu bröckeln. Der mit dem schleichenden Entzug der Konzession einhergehende Verlust des Prestiges der alemanes könnte einer der Motoren für die brutale Enthauptung Huttens und seiner Gefährten durch Juan de Carvajal gewesen sein. Für Denzer (vgl. 2005) sagt der Entzug der Konzession an die Welser 
allerdings nichts über das Verhältnis der Krone zur Handelsgesellschaft aus. Er stellt ihn vielmehr in den größeren Zusammenhang der Entmachtung aller Machthaber in den neu eroberten Ländern des Imperiums, die die Krone mit den Leyes Nuevas 1542 eingeleitet hatte.

Die Einbettung der Studie in die Debatten der Kolonialforschung wird in Kapitel 2 und 3 vorgenommen. Diese hat sich in erster Linie mit den Texten der >Entdeckung/Eroberung Amerikas, also mit Reiseliteratur, beschäftigt. Hier wurde die Forschungslücke ausgemacht, an der die vorliegende Studie angesiedelt ist: Sie beschäftigt sich mit Texten, die im Anschluss an die ersten Phasen der >Entdeckung und Eroberung, nämlich am Übergang zur Kolonisierung und Herstellung von kolonialer Normalität, produziert wurden. Grundlegend wurde die Hypothese aufgestellt, dass die einzelnen Phasen, die die europäische Expansion gliedern (`Entdeckung`, Eroberung und Kolonisierung), mit der Produktion von Texten spezifischer Ausprägung verbunden ist. Während für die ersten beiden Phasen die Textsorte »Reisebericht« im Vordergrund stehen dürfte, verbreitert sich das Spektrum der Textsorten in der Dritten, wobei gegebenenfalls auch von einer verstärkten Verwischung der Textsorten gesprochen werden kann. Dies zeigt sich zum Beispiel im Textkonglomerat "Ain andere Histori/von newlich erfundenen Jnseln der Landtschafft Jndie« - »Reisebericht« beziehungsweise »Brief - oder in den Prozessakten, wenn sich die Zeugenaussagen, eine genuin juristische Textsorte, streckenweise der Textsorte »Reisebericht« annähern.

Ein anderer Zweig innerhalb der Kolonialforschung beschäftigt sich mit der Funktion des Schreibens als koloniales Machtinstrument. In einem zweiten Teil des Kapitels werden die entsprechenden Begriffe »bürokratischer Apparat «, »Interpellation « und »koloniales Subjekt« entwickelt. Die Autoren dieser Richtung zeigen auf, wie sich die Europäer das sentdeckte Land schreibend aneigneten. Dazu gehörte die Einschreibung der von ihnen (um)benannten Orte in Landkarten und Expeditions- und Reiseberichte, aber auch die Möglichkeit, die Botschaften durch Zeit und Raum frei bewegen zu können. Ganz grundsätzlich ermöglichte die Alphabetschrift, eine Auslegeordnung vorzunehmen, sei es mit einer Karte eines zu erobernden Landes oder mit der Konzipierung und Planung von neu zu gründenden Städten. Die Texte unseres Korpus zeigen verschiedene Ausprägungen dieser Funktionen von Schrift. Diese werden in den einzelnen Kapiteln konkretisiert.

Kapitel 4 setzt sich aus den Analysen des Textkonglomerats »Ain andere Histori/von newlich erfundenen Jnseln der Landschafft Jndie « und der Briefe, die Philipp von Hutten aus Venezuela geschrieben hatte, zusammen. Diese 
beiden Texte liegen der Schwelle des Übergangs von der Eroberung zur Kolonisierung am nächsten: Die Briefe sind Zeugnis und Vollzug der Eroberung der Provinz Venezuela durch Hohermuth von Speyer und später durch Philipp von Hutten, sind also Texte der >Entdeckung/Eroberung, während sich die »Inseln« eher als Text der Kolonisierung zu lesen geben, indem sie sich an ein breiteres Publikum wenden und - wie die eingefügten Zwischentitel prägnant zu lesen geben - rückblickend im Namen des Kaisers vollzogene >Entdeckungen und Eroberungen verzeichnen, dem Imperium zuschreiben und dieses somit konsolidieren.

Die beiden Texte wurden zunächst vergleichend untersucht. Dies war nötig, um die Bezüge zwischen beiden zu klären: Die »Jnseln« - bereits im Jahr 1550 im Anhang zur deutschen Übersetzung des zweiten und dritten Briefes von Hernán Cortés an Kaiser Karl V. veröffentlicht - wurden vom Drucker Philipp Ulhart aus Versatzstücken einzelner Hutten-Briefe zusammengestellt und stimmen in diesen Teilen wörtlich mit ihnen überein. Die typischen Anrede- und Grußformeln, die Briefe als solche markieren, wurden bis auf wenige Ausnahmen entfernt, und ganze Passagen wurden umgeschrieben, weggelassen oder verschoben, andere wurden hinzugefügt. Der Name des Autors, Philipp von Hutten, wurde getilgt. Die »nseln« wurden der deutschen Übersetzung eines Briefes des spanischen Chronisten Gonzálo Fernández de Oviedo vorgeschaltet, wodurch der Eindruck entstand, der ganze Text stamme von Oviedo. Diese Manipulationen führten in ihrer Gesamtheit zu teilweise unauflösbaren inhaltlichen Brüchen, was den Drucker und offenbar auch die Leserschaft nicht weiter zu stören schien. Möglicherweise wurden sie durch die Anbindung an das literarische Genre der »Insulae« - dessen vielleicht prominentester Vertreter Morus' Text Utopia ist - aufgefangen, das zu jener Zeit weit verbreitet waren. Das Genre zeichnet sich durch den Entwurf von Sozialutopien aus, die gewöhnlich auf Inseln, abgetrennt von der >Welt<, verortet werden. Die Texte des Genres sind von der grundlegenden Ambivalenz geprägt, die jede Insel kennzeichnet: dass sie sich nämlich von einem großen Ganzen abhebt und durch diese Abkoppelung gleichzeitig mit ihm verbunden ist. Ohne das große Ganze ist die Insel keine Insel. Wie Balasopoulos (vgl. 2008) für Utopia herausgearbeitet hat, kann der Text einerseits eine geographische Insel beschreiben und eine Utopie entwerfen, die als Gegenentwurf zum großen Ganzen mit diesem verbunden ist. Andererseits kann der Text aber auch selbst für eine >Diskursinsel stehen, die sich vom großen Ganzen der beschreibenden Diskurswelt abhebt und gleichzeitig mit ihr gekoppelt ist. 
Liest man nun den Text der »Jnseln« als Vertreter des Genres der »Insulae«, beschreibt er einerseits die Welt und entwickelt andererseits einen Gegenentwurf, einen Gegenraum zu der Welt. Die Beschreibung der Welt erfolgt dadurch, dass der Text die Inseln benennt, die die »Hispanier« bereits in Besitz genommen haben, und damit als Besitz festschreibt; andererseits erschreibt er einen mentalen Raum namens »Venaßla« (Venezuela), indem das wohl durch den Drucker Ulhart zusammengezimmerte Ich die Topographie des Landes entlang der Expeditionen durch das Hinterland von Coro beschreibt, die Ortsnamen und die Namen der Völker nennt und es damit mental, in den Köpfen der Europäer, als neuen Teil des Imperiums der europäischen Expansion erschließt. Venezuela ist zwar keine Insel im geographischen Sinne wie Utopia; metaphorisch liegt das Land jedoch durch die wilden und bisweilen gefährlichen Wasser des Ozeans - Sinnbild des Flusses, mit dem Utopus Utopia vom Festland getrennt hatte - von der Heimat abgekoppelt.

Philipp von Huttens Briefe, in ihrer Gesamtheit erst seit 1999 in einer Publikation zugänglich, wurden in diesem Buch einzeln dargestellt und in ihrer spezifischen >kolonialen Situation verortet. Sie sind, entgegen ihrem vordergründig privaten Charakter, >koloniale Texteく, weil sie in einer `kolonialen Situation entstanden sind und gleichzeitig zur Kolonialisierung beigetragen haben. Hutten hatte sich in und durch seine Briefe den Raum des Hinterlandes von Coro schreibend angeeignet, indem er - immer schon als legitimer und legitimierter Eroberer - Geographie, Topographie, Klima und Völker des Territoriums beschrieb. Sein Schreiben fütterte das koloniale Archiv, das damit diesen Prozess nicht nur dokumentierte, sondern auch vollzog: In der >Kontaktzone`seiner Briefe wurde die (Nicht-)Einschreibung der Stimme der >Anderen verhandelt.

Durch das Zur-Schau-Stellen der eigenen Fähigkeiten und Vorzüge will sich Hutten Zugang verschaffen zum bürokratischen Apparat. Sein Mittel ist eine Berichterstattung, die sowohl auf Vollständigkeit seiner Taten (Archiv) als auch auf die Erschaffung eines Monuments abzielt. Diese beiden Ziele widersprechen sich: Vollständigkeit in der Darstellung birgt die Gefahr der Eintönigkeit und steht einem heroisierenden Hervorheben von leicht memorierbaren Einzeltaten als Eroberer entgegen. Vielleicht liegt das Sperrige, das Huttens Briefe kennzeichnet, (auch) in diesem Widerstreit begründet. Huttens Schreiben ist gezeichnet von Spannungen zwischen erhofftem, erwartetem und ausgebliebenem Erfolg. Das Monument Philipps von Hutten wird nach seinem Tod durch andere Texte geschaffen: zum Beispiel durch Diego 
Ruiz de Vallejos Brief, der ihn zur >Lichtgestalt $<$ modelliert, welche die spanischen Chronisten aufgenommen haben.

Obwohl die Liste der Adressaten, an die sich die Briefe wenden - Freunde, Familienangehörige - , suggerieren mag, dass die Briefe tendenziell >privater Natur sind und ein identifizierbares $>$ Ich < hervorbringen, legt eine genauere Untersuchung nahe, dass Huttens Schreiben letztlich als Ergebnis der Mechanismen des bürokratischen Apparats zu lesen ist. Wie die anderen Texte des Korpus auch verkörpern die Briefe die stereotype Vision des kolonialen Subjekts das einen stereotypen Diskurs produziert, der nach Rolena Adorno die Werte der maskulinen und christlichen Kultur repräsentiert (vgl. Adorno 1988b: 56). Die »andere Histori/von newlich erfundenen Jnseln der Landtschafft Jndie« verlässt den >privaten Rahmen der (zumindest an der Textoberfläche) persönlich gehaltenen Briefe und richtet sich durch die Veröffentlichung in Buchform an ein breiteres Publikum. Der Text ist insofern ein Produkt des bürokratischen Apparats, als er sich an die zu Hause gebliebenen Europäer und Europäerinnen richtet, diese - um mit Mary Louise Pratt zu sprechen - in das "globale Projekt« (Pratt 2008: 3), das sich heute europäische Expansion nennt, einbindet und damit zu kolonialen Subjekten macht.

Kapitel 6 beschäftigt sich mit den Akten der Prozesse des Juicio de Residencia - einem Amtsprüfverfahren zur Amtsführung der Welser - und dem Kriminalprozess gegen Juan de Carvajal. Die Akten beider Prozesse dokumentieren die Prozesse nicht nur, sondern bilden auch eine >Kontaktzone ‘; indem sie einen normalen >Alltag< schaffen, vollziehen sie in prominenter Weise den Übergang zur Installation und Festigung des Imperiums.

Der Begriff koloniales Subjekt suggeriert, dass sich damit eine interpellierte `Stimme< verlauten lässt. Dies trifft auf Briefe $\mathrm{zu}$; bezüglich der Prozessakten ist die Verwendung dieses Terminus unangemessen, weil an deren Produktion viele Hände, Interessen, Techniken und Mechanismen mitgewirkt haben, die den Text in seiner Schlussgestalt maßgeblich formten. Daher spreche ich in diesem Zusammenhang anstelle des kolonialen Subjekts von einem kolonialen Subjektgefüge. Dieser Ausdruck impliziert ein ganzes Bündel von Merkmalen, die die Aussagekraft der Akten betreffen. Um diese einer fruchtbaren Lektüre zu unterziehen, musste der Text auf Auffälligkeiten hin untersucht werden. Diese können zu Ankerpunkten werden, an denen sich weitere Überlegungen anschließen lassen. Eine dieser Auffälligkeiten besteht beispielsweise darin, dass einzelne Zeugenaussagen wortwörtlich übereinstimmen. Da es unmöglich ist, dass einzelne Zeugen wörtlich die gleichen Aussagen machen, gerät die Arbeitsweise des Schreibers in den Blick: Wenn 
er einzelne Aussagen mehrmals an verschiedenen Orten einfügt, könnte dies entweder auf seine mangelhafte Fähigkeit, eine nachlässige Arbeitsweise oder aber auf eine spezielle Rechtskultur hinweisen. Auf jeden Fall muss dieser Fakt bei der Lektüre stets mitbedacht werden. So lassen sich in letzter Konsequenz die `Zeugenaussagen ligen Zeugen noch an die tatsächlich getätigte Aussage zurückbinden. Ein weiteres Problem besteht darin, dass die Notare vorgefertigte Formeln und Formulierungshilfen einzusetzen pflegten, die sie zirkulierenden Manualen entnahmen. Diese Formeln konnten die Texte in Extremfällen bis zur Unverständlichkeit verunstalten, wenn sie zu exzessiv verwendet wurden. Dazu kommt, dass die Formulierungshilfen in den Manualen teilweise so gestaltet waren, dass sie ganze >Plots« mitlieferten, von denen die Schreiber bei der Anwendung im konkreten Fall natürlich abstrahieren mussten. Die Manuale konnten also nicht nur auf der Ebene des sprachlichen Materials, sondern auch auf inhaltlicher Ebene in die Akten einfließen. Für heutige Lesende ist der Grad dieses Einflusses kaum zu bestimmen.

Die Lektüre der Akten der Residencia und des Kriminalprozesses hat gezeigt, dass in den Prozessen keine Wahrheitsfindung im modernen Sinne stattfand. Der Richter Juan Pérez de Tolosa hatte den Angeklagten Juan de Carvajal schon vor Beginn der beiden Prozesse gegen ihn öffentlich des Verbrechens beschuldigt, Hutten und Welser böswillig und ohne Legitimation hingerichtet zu haben. Alle danach eingeholten Zeugenaussagen bestätigten diese Vorverurteilung. Die Fragen, die Carvajal am Schluss der Erkundungen vorgelegt wurden, zurrten die genannte >offizielle Version der Ereignisse fest. Sie setzen sich zusammen aus allen möglichen Anschuldigungen, die von verschiedenen Quellen stammen mussten. Sie sind zwar als Fragen formuliert, wurden aber nicht als solche intendiert: Nicht Carvajals Antworten, sondern die Fragen selbst lieferten die von Richter Tolosa gewünschten Informationen. Carvajal blieb nur der Part, sie zu bestätigen oder abzustreiten; allerdings stießen seine Aussagen auf jeden Fall ins Leere. Es wäre zumindest denkbar, dass ohne Tolosas öffentliche Vorverurteilung andere Versionen zur Sprache gekommen wären. $\mathrm{Zu}$ bedenken ist nicht nur, dass die Prozesse nicht unbedingt vorrangig zum Ziel hatten, eine verborgene >Wahrheit ans Licht zu bringen; ebenso wichtig war die Bildung von Einheit unter den Einwandernden, die in vielen Fällen eine Art Zufallsgemeinschaft bildeten und zusammengeschweißt werden mussten.

Kapitel 6 befasst sich mit drei Briefen und einem königlichen Mandat, das als Antwort auf den dritten Brief gelesen und deshalb ins selbe Kapitel ein- 
geordnet wird. Der erste Brief stammt von Diego Ruiz de Vallejo, der zweite von Galeotto Cei, einem florentinischen Handelsreisenden, und der dritte von Richter Juan Pérez de Tolosa. Diego Ruiz de Vallejo, der von der Truppe Carvajals zu Hutten übergelaufen war, verfasste seinen Brief noch während des Kriminalprozesses gegen Carvajal. Er richtet sich darin an einen gewissen, nicht näher identifizierten "muy noble señor «, den er über die schwierigen Verhältnisse in der Provinz unterrichtet, damit dieser informiert sei. Indem er die Informationen, die er als Augenzeuge gewonnen hat, gezielt weiterleitet, tritt er als Organisator der Kolonisierung Venezuelas auf - wenn auch im Hintergrund. Sein Brief ist also, ähnlich wie diejenigen Huttens, keineswegs >privater Natur. Als »kolonialer Text « betreibt er die Marginalisierung der »indios«: Treten diese bei Hutten hier und da, meist flüchtig in Erscheinung, nehmen sie bei Vallejo als den Kolonialherren Dienende einen fixen schmalen Raum am Rande des aufgespannten Schauplatzes ein.

Selbstverständlich ist auch der Bericht Galeotto Ceis als »kolonialer Text« zu lesen, obwohl weder der Verfasser noch der Adressat direkten Zugang zum bürokratischen Apparat haben. Das Schreiben schafft eine Differenz zwischen den Europäern und den indigenen Gemeinschaften und weist Letztere als unterlegen aus. Ceis Relazione unterschiedet sich aber in anderer Hinsicht von den anderen: Er ist geprägt von literarischen florentinischen Traditionen, insbesondere der beffa (Spott). Für die anderen Texte des Korpus heißt das umgekehrt, dass sich ihr Schreiben den literarischen Traditionen verschloss, oder zumindest, dass das juristische Schreiben andere denkbare Möglichkeiten des Schreibens verdrängte. Diese Folgerung schließt an die oft zitierte Aussage von González Echevarría an, dass die erzählende Literatur auf das juristische Schreiben zurückging und das Schreiben im 16. Jahrhundert den Gesetzen untergeordnet gewesen sei: »En el siglo XVI, escribir estaba subordinado a la ley. [...] La narrativa, tanto novelesca como histórica, se derivó de las formas y regulaciones de la escritura jurídica« (González Echevarría 2000: 77). Nicht nur Ceis Text bildet in gewisser Weise einen sSonderfalk, sondern auch die Forschungsliteratur, die sich mit Ceis Relazione befasst: Sie versucht in erstaunlich unkritischer Weise, an die sgroßen spanischen >Entdeckungen anzuschließen.

Juan Pérez de Tolosa, der Richter des Juicio de Residencia zu den Welsern und des Kriminalprozesses gegen Carvajal, richtet seinen Brief einige Wochen nach Vollstreckung des Urteils an den König. Er enthält einen ausführlichen Bericht über sein Handeln während des Prozesses. Die Verhältnisse in der Provinz stellt er als besonders prekär dar, wie um damit sein Vorgehen 
zu rechtfertigen, das ihm viel Einsatz und Investition abverlangte. Im zweiten Teil des Briefes verhandelt er denn auch handfest über die Art und Höhe seiner Entlohnung, die er vom Hof erwartet. Eine direkte Antwort des Königs auf diese Eingabe liegt meines Wissens nicht vor, wohl aber eine real cédula, in der Karl V. den Indienrat auffordert, den Kriminalprozess wieder aufnehmen zu lassen und Tolosa anzuweisen, auch die übrigen an der Enthauptung Huttens und Welsers Mitschuldigen einer gerechten Strafe zuzuführen. Diese Anweisung zielt inhaltlich am Schreiben Tolosas vorbei: Dieser hatte darin die alleinige Verurteilung Carvajals explizit damit gerechtfertigt, dass die Beteiligten durch die Vorkommnisse geeint worden seien und dass nun Ruhe in die Provinz einkehren müsse. Auffällig ist auch, dass die real cédula ein Bild von Philipp von Hutten entwirft, das von dem in den anderen Texten konstruierten abweicht: das Bild eines großen Entdeckers reicher Provinzen. Obwohl die cédula aus der Feder des obersten Machthabers stammt und eine sehr eingängige, Monument kreierende Version des >Falls« Hutten-Carvajal liefert, geht sie aber erstaunlicherweise nicht in die Geschichte ein. Macht scheint kein Garant dafür zu sein, sich nachhaltig Gehör zu verschaffen. Möglicherweise wurde das königliche Mandat für die spätere historiographische Aufarbeitung als weniger sauthentisch eingestuft als die Augenzeugenberichte, weil es räumlich und auch zeitlich am weitesten vom Tathergang entfernt ist. Das Zentrum der Macht selbst treibt die europäische Expansion an und tritt als kolonisierendes Subjekt auf. Trotzdem scheint es Abhängigkeiten unterworfen zu sein, denen es im Text nachgibt. Das Mandat gibt Anweisungen, die mit den Verhältnissen in Venezuela zum Zeitpunkt des Schreibens nicht mehr vereinbar waren, und blieb damit ohne Folgen. Daraus konnte dem König aber auch kein Schaden erwachsen, und er konnte ohne Risiko den Forderungen der Familien der Hutten und Welser nachkommen, zumindest auf dem Papier. Mit dem Mittel der Schrift kann wenigstens ein Teil der Ansprüche bedient werden. »Papier ist geduldig« - die Redensart gilt hier vielleicht in ganz besonderer Weise. Was von Huttens Bild geblieben ist, ist die bei Vallejo angelegte, aber auch bei anderen Texten fortgesetzte Modellierung als >Lichtgestalt<, die den Gegenpart zur Monstrosität Juan de Carvajals bildet. Dass Hutten ein Repräsentant der von Tolosa - und nicht nur von ihm - verteufelten Welser war, tat der Verfestigung keinen Abbruch.

In allen Texten konstituieren sich koloniale Subjekte auf folgende Weise: Sie präsentieren die Europäer als den Einheimischen überlegen und rechtfertigen damit direkt oder indirekt die Eroberung; alle betreiben sie eine Art sSelbstinszenierung<, die das eigene Handeln rechtfertigt oder in ein gutes 
Licht rückt und die eigene Position als Informationslieferant festschreibt. Diese muss gegen eventuell konkurrierende Informationen und Informationsquellen gesichert werden. Philipp von Huttens Texte beschreiben die »Indier « als grundsätzlich anders: mal als Nackte, Wilde, Kannibalen, mal als potentielle Bedienstete und Lastenträger, nach denen die »Christen« jagten, mal als »befriedet«, zuverlässig, die Ernährung der »Christen« sichernd. Genau umgekehrt - nämlich durch Verschweigen oder Gleichmachen - vollzieht sich die Konstituierung des kolonialen Subjektgefüges in den Prozessakten: Die Akten des Juicio de Residencia blenden die Andersartigkeit der Indigenen textuell aus, indem die neuen Machthaber selbstverständlich über das fremde Territorium verfügen und es verwalten, als ob es immer schon ihnen gehört hätte; die Akte des Kriminalprozesses homogenisiert die Zeugen »Magdalena« und »Perico« durch die Namen textuell und verleibt sie dem Diskurs des kolonialen Subjekts ein. Einziges Indiz der Andersartigkeit bildet das Wort »indios«, eine Bezeichnung, die nur durch einmaliges Aussprechen den ganzen Prozess der `Entdeckungく, Eroberung und Kolonialisierung ablaufen lässt.

Zur kolonialen Subjektbildung in den Briefen der drei Figuren Diego Ruiz de Vallejo, Galeotto Cei und Juan Pérez de Tolosa lässt sich Folgendes feststellen: Vallejo, als Briefschreiber offenbar geübt in der Teilnahme an Vorgängen der kolonialen Justiz- und Verwaltungsprozesse der Provinz, agiert als koloniales Subjekt, indem er sich einerseits Richter Tolosa und seiner Stoßrichtung unterwirft und andererseits die »indios« marginalisiert und ihnen textuell kaum Raum gewährt. Obwohl Galeotto Cei unter etwas anders gelagerten kulturellen Einflüssen und mit mehr Freiheiten als die Spanier schreibt, manifestiert auch sein Schreiben ein koloniales Subjekt, indem es mit der Differenz zwischen Kolonisierenden und Kolonisierten operiert und diese ausweist. In diesem Fall liegt vielleicht nicht die prototypisch koloniale Situation vor, weil die italienischen Reisenden beziehungsweise Schreibenden weniger stark an institutionelle Vorgaben des bürokratischen Apparats gebunden waren. Trotzdem muss auch die Relazione als »kolonialer Text« - wenn auch in etwas anderer, merkantilistischer Ausprägung - bezeichnet werden. Juan Pérez de Tolosa verkörpert als Magistrat und damit per se Angehöriger des bürokratischen Apparats hingegen das koloniale Subjekt schlechthin. Er unterwirft sich in seinem Schreiben dem König als kolonisiertes Subjekt; gleichzeitig beteiligt er sich mit seinem Schreiben an der Eroberung und Ausbeutung der Provinz als kolonialisierendes Subjekt.

Für die Texte des Korpus dieser Studie hat sich gezeigt, dass das koloniale Schreiben, das in ihnen zur Anwendung kommt, untrennbar mit der 
Konstituierung eines kolonialen Subjekts beziehungsweise eines kolonialen Subjektgefüges verbunden ist. Dabei sind zwei Prozesse zu beobachten: erstens das Operieren mit der Differenz zwischen Kolonialisierenden und Kolonialisierten durch explizites und distanzierendes Beschreiben, Marginalisieren oder Verschweigen des `Anderen<, zweitens die Selbstinszenierung vor dem Hintergrund der alles Schreiben prägenden »economy of mercedes« (Folger 2011: 16), die der Sicherung der eigenen Position als unverzichtbare Informationsquelle und der Rechtfertigung des eigenen Handelns dient. Diese Prozesse sind Teil des bürokratischen Apparats, der die Mechanismen, Muster und Schablonen für die Textgenerierung liefert.

Die Grenzen zwischen dem, was in diesem Buch koloniales Subjekt beziehungsweise Subjektgefüge genannt wird, verlaufen fließend, wie sich in den Textanalysen immer wieder gezeigt hat. Obwohl die beiden Begriffe ähnlich gelagert sind, konnte die Verwendung beider fruchtbringend eingesetzt werden. Eine Ersetzung des Begriffs »koloniales Subjekt« durch »koloniales Subjektgefüge« würde gewisse Nuancen und Schattierungen ausradieren, weil sie gewissermaßen aus dem Fokus fallen würden.

$>$ Koloniale Texte sind keine transparenten Fenster, die unmittelbare Einsicht in die Vergangenheit gewähren. Im Gegenteil: Ihre Lektüre verlangt ein ganzes Prozedere, das die Dokumente zum Sprechen bringt. Die skoloniale Kommunikation hat uns zwar tausende und abertausende Dokumente hinterlassen. Wir haben in der Untersuchung der Texte jedoch gesehen, dass ihre Lektüre einerseits einige Fragen beantwortet, andererseits aber mindestens ebenso viele Fragen aufwirft. >Koloniale Texte liefern komplexe Bedeutungsangebote, die nicht alle auf den ersten Blick erkennbar sind und erst herausgearbeitet werden müssen. Dabei zeigt sich, dass die sich in den Texten konstituierenden kolonialen Subjekte in ihrer sowohl kolonialisierenden als auch kolonialisierten Ausprägung das Unternehmen der europäischen Expansion getragen haben. Schreiben und Unterwerfen gehören im kolonialen Kontext untrennbar zusammen, indem das koloniale Schreiben die Konstituierung eines dichten Netzes kolonialer Subjekte - eines Subjektgefüges - überhaupt erst ermöglicht. 
